\title{
Cadmium Zinc Telluride based Infrared Interferometry for X-ray Detection
}

\author{
A. Lohstroh, ${ }^{1 \text { a) }}$ I. Della Rocca, ${ }^{1}$ S. Parsons,${ }^{1,2}$ A. Langley, ${ }^{2}$ C. Shenton-Taylor, ${ }^{2}$ \\ D. Blackie $^{2}$ \\ ${ }^{1}$ Department of Physics, University of Surrey, Guildford GU2 7XH, United Kingdom \\ ${ }^{2}$ AWE Aldermaston, Reading, RG7 4PR, United Kingdom
}

\section{Abstract}

Cadmium Zinc Telluride (CZT) is a wide band gap semiconductor for room temperature radiation detection. The electro-optic Pockels effect of the material has been exploited in the past to study electric field non-uniformities and their consequence on conventional detector signals in CZT, by imaging the intensity distribution of infrared (IR) light transmitted through a device placed between crossed polarizers. Recently, quantitative monitoring of extremely high intensity neutron pulses through the change of transmitted IR intensity was demonstrated, offering the advantage to place sensitive electronics outside the measured radiation field. In this work, we demonstrate that X-ray intensity can be deduced directly from measuring the change in phase of $1550 \mathrm{~nm}$ laser light transmitted through a $7 \times 7 \times 2 \mathrm{~mm}^{3}$ CZT based Pockels cell in a simple Mach Zehnder interferometer. X-rays produced by a $50 \mathrm{kVp}$ Mo X-ray tube incident on the CZT cathode surface placed at $7 \mathrm{~mm}$ distance cause a linearly increasing phase shift above $0.3 \mathrm{~mA}$ tube current, with $1.58 \pm 0.02 \mathrm{rad}$ per $\mathrm{mA}$ for an applied bias of $500 \mathrm{~V}$ across the $2 \mathrm{~mm}$ thick device. Pockels images confirm that the sample properties are in agreement with literature, exhibiting electric field enhancement near the cathode under irradiation, which may cause the non-linearity at low X-ray tube anode current settings. The laser used to probe the X-ray intensity causes itself some space charge, whose spatial distribution does not seem to be 
exclusively determined by the incident laser position, i.e. charge carrier generation location, with respect to the electrodes.

${ }^{\text {a) Email: A.Lohstroh@ @urrey.ac.uk }}$ 


\section{Introduction}

Cadmium Zinc Telluride (CZT) is a well-established semiconductor material for spectroscopic ionizing radiation detection (Ref.1) with application areas ranging from national security, medical imaging, astronomy and beyond (see for example Ref. 2, 3, 4, 5). Due to its cubic crystal structure, an ideal CZT crystal is expected to have isotropic optical properties that do not exhibit birefringent behavior. However, birefringence can occur when the cubic symmetry is broken due to strain associated with intrinsic extended defects or by electric fields present in the material (for example dislocations (Ref 6)) which cause a relative shift in lattice positions between Cd (Zn) and Te lattice sites. The latter effect is referred to as the Pockels' effect, and can also be expressed as a change in refractive index $n$ with electric field strength $E$. The transmitted light intensity $I_{\text {trans }}$ in the used geometry can be described by (Ref.7)

$$
I_{\text {trans }}=I_{\max } \sin ^{2}\left(\frac{\sqrt{3} \pi n_{0}^{3} r_{41} l}{2 \lambda} E\right)
$$

Where $I_{\max }$ is the maximum transmitted intensity (corresponding to a phase of $\pi / 2$ ), $r_{41}$ the Pockels electro-optic coefficient, $n_{0}$ the field free refractive index at wavelength $\lambda$ and $l$ the path length of the light through the crystal. Many research groups have exploited the effect in order to study the changes in the electric field profile of the material as a function of temperature and irradiation and illumination conditions (see for example Ref. 8, 9, 10,11, 12). These studies have shown that planar CZT devices exhibit an increase in electric field near the cathode at room temperature under near IR illumination and/or X-ray irradiation and a collapse of electric field in the majority of the device due to space charge build up. More recently, Nelson et al. (Ref. 13, 14, $15,16)$ have demonstrated that neutron induced changes in transmission of polarized laser light through biased crystals (due to the radiation induced changes in the birefringence) can be 
exploited to measure the intensity of extremely high flux reactor neutron pulses and constant reactor beam port irradiation, environments where traditional detector technology saturates. This approach of using ionizing radiation induced changes in optical properties as a radiation detection mechanism offers the opportunity to place the radiation sensitive detection medium at a long distance from the radiation field combined with in-situ read-out. This can be particularly advantageous in high intensity fields where radiation induced damage in the electronic components is a significant consideration. On the other hand, the aforementioned space charge build up under irradiation also implies non-uniform changes in refractive index and hence the development and understanding of such detector systems is yet at an early stage.

In this work, we demonstrate X-ray intensity measurements using a CZT crystal with a simple sandwich electrode structure within a Mach Zehnder Interferometer. The proposed system measures the phase shift $\phi$ of the transmitted light directly rather than its intensity which is given by (Ref. 7). $\phi=\frac{\sqrt{3} \pi n_{0}^{3} r_{41} l}{\lambda} E(2)$

\section{Sample characterization through transmission intensity measurements}

The $7 \times 7 \times 2 \mathrm{~mm}^{3}$ CZT material, synthesized by the vertical Bridgeman technique, has been produced by Yinnel Tech Inc, and polished at the University of Surrey as described in Ref. 17. Gold electrodes of $90 \mathrm{~nm}$ thickness and $6 \times 6 \mathrm{~mm}^{2}$ area, were deposited by sputtering onto two opposite surfaces separated by the $2 \mathrm{~mm}$ thick CZT sample. Preliminary characterization using the Pockels imaging system and the same sample, following removal, polishing and redeposition of the electrical contacts as described in Ref. 9 was carried out at room temperature, which yielded results consistent with those described in Ref 8 . The light intensity used for the 
image acquisition is typically kept below $1 \mathrm{~mW} / \mathrm{cm}^{2}$ in order to avoid perturbations of the electric field by the probing light source. The device shows relatively uniform light transmission at $\lambda=980 \mathrm{~nm}$, which implies a uniform electric field profile. An example image taken at 500 Volt applied bias $V$ can be seen in Figure 1. The slightly darker edges observed at the $2 \mathrm{~mm}$ long sides of the crystal are caused by a reduction of field strength at the crystal sides, similar to those observed by Yang et al. (Ref 18).

The average transmitted intensity extracted from the Pockels' images as a function of $V$ follows qualitatively the expected quadratic sinusoidal shape of equation (1). The fit of the data indicates that maximum transmission is predicted for $1026 \pm 19 \mathrm{~V}$ and implies that the optimum applied voltage for the Pockels cell operation (i.e. where small electric field changes yield the largest change in transmission) is at $513 \pm 10 \mathrm{~V}$, close to the $500 \mathrm{~V}$ value shown in Figure 1. This value matches the data reported in Ref.13, reaching maximum transmission at approximately $1 \mathrm{kV}$ applied bias across the $2 \mathrm{~mm}$ thick CZT sandwich structure; the small variation in maximum voltage is in agreement with the slight difference in optical path lengths in the two experiments. However fitting the intensity data using the refractive index of CZT $n_{0}=2.8$ (Ref.19,20), $\lambda=980$ $\mathrm{nm}, l=6.0 \pm 0.3 \mathrm{~mm}, d=2.0 \pm 0.1 \mathrm{~mm}$ results in a value of $r_{41}=8.4 \pm 0.6 \times 10^{-12} \mathrm{~m} / \mathrm{V}$, which is above the typically quoted value for undoped CdTe and CZT at $\lambda \approx 1000 \mathrm{~nm}$ between 4.5 and $5.5 \times 10^{-}$ ${ }^{12} \mathrm{~m} / \mathrm{V}$ (Ref. 20, 7). Shwartz et al. report dramatically increased electro-optic coefficients from $5.5 \times 10^{-12} \mathrm{~m} / \mathrm{V}$ in undoped CZT up to $900 \times 10^{-12} \mathrm{~m} / \mathrm{V}$ in Vanadium doped crystals (Ref. 8) at $\lambda$ $\approx 1000 \mathrm{~nm}$, hence it might be possible that the discrepancy is caused by intentional or unintentional impurities in the material.

A $1550 \mathrm{~nm}$ infrared (IR) laser (Thorlabs LDM1550, $4.5 \mathrm{~mW}$ ) was chosen for the interferometer study due to its easy availability and compatibility with mainstream fibre optic technology; 
approximately $55 \%$ of the laser light will be transmitted through the sample at this wavelength (Ref.12). Pockels images have also been acquired with the IR laser beam passing through the sample perpendicular to the optical axis of the Pockels microscope as indicated in Fig. 1. The intensity cross sections shown as a function of distance from the cathode reveal that the additional IR laser light will cause some electric field enhancement under the cathode within the sample - as illustrated in the Pockels image in Figure 1 and the intensity profiles in Fig. 2 a). The intensity distribution under opposite bias polarity is not perfectly symmetric, however there is clear enhancement in transmission near the cathode in both cases. The imperfect symmetry as well as the field enhancement under the cathode under IR illumination are in agreement with studies reported in the literature that used $950 \mathrm{~nm}$ light emitting diodes as additional illumination source. (Ref. 10, 11, 12). The laser illumination was subsequently limited by a $0.1 \mathrm{~mm}$ slit and the laser slit combination moved across the sample surface, while maintaining a fixed Pockel's camera view. For laser-slit combination heights smaller than $2.1 \mathrm{~mm}$ and larger than $4.3 \mathrm{~mm}$, the laser does not actually hit the sample and hence the black intensity profiles shown in Fig. 2 b) display the same shape as the ones without illumination shown in Fig. 2 a) at the same bias condition. For heights between 2.3 and $4.1 \mathrm{~mm}$, the laser is incident on the $2 \mathrm{~mm}$ thick sample (partially or completely, depending on the exact position) and the intensity profiles show enhancement near the cathode for all positions. This demonstrates that the space charge build up position is not purely determined by the incident laser position, i.e. the free charge carrier generation location. The slightly lower maximum value of the profiles compared to the one shown in Fig 2. a) for the full laser illumination, is caused by the reduction in laser intensity due to the collimation.

\section{The Interferometry set-up}


For the interferometry measurements, the IR laser, filtered by a linear polarizer was incident on a beam splitter at approximately $45^{\circ}$. Both beams encounter subsequent mirrors at $45^{\circ}$ before being recombined at the second beam splitter, as indicated in Fig. 3. The intensity of the recombined signal is monitored with an InGaAs photodiode (Thorlabs PDA10CS), whose output was acquired using a Tektronix TDS 3012 digital oscilloscope. A phase retarder (Thorlabs LCC1111C) was inserted into one arm of the split beam path and the CZT sandwich structure in the other. The linear polarizer in front of the laser was aligned parallel to the applied electric field and the laser beam width was not limited by a collimator in order to exploit the full available signal intensity. The system was highly sensitive to vibrations, and changes in observed photodiode output were seen without applied bias or X-ray irradiation. These correlate with minute changes in alignment due to the changes in optical path lengths and, potentially, light guide effects caused by the CZT crystal geometry.. The maximum and minimum photodiode output levels, $A_{\max }$ and $A_{\min }$, correspond to completely constructive and completely destructive interference of the recombined beam. These levels were established before and after each measurement by inducing small mechanical vibrations in the system by tapping the mounting board, illustrated in Figure 4. Small deviations in the upper and lower limit levels observed before and after the signal data (i.e. with X-rays/bias switched on) might have been caused by slight changes in the overlap of the recombined beams due to the changes in beam direction caused by the refractive index changes, when switching the bias/X-ray generator on and off. The photodiode signal amplitude change induced by changes in the applied bias and/or X-ray intensity took typically up to 2 seconds to stabilize. In the case of switching X-ray irradiation, this is similar to the time it takes for the Xray tube to switch from no output to maximum output, which is also between 1 and 2 seconds. 
The amplitude levels $A$ and $A^{*}$ before and after the change in experimental parameter, respectively, were converted into phase values using:

$$
\varphi=\arcsin \left(2 \frac{A-A_{\min }}{A_{\max }-A_{\min }}-1\right)
$$

Due to the non-linear, relationship between the photodiode signal amplitude and the phase shift detected, $\phi=\left(\varphi^{*}-\varphi\right)$, the uncertainties of the extracted phase shifts $\phi$ do not only depend on the stability of the raw data, but also on the phase value of the start and end positions. The analysed

photodiode signal traces did not include signals that transited through $A_{\max }$ or $A_{\min }$. Ten independent measurements have been taken at each setting in order to achieve similar statistical significance across all data sets.

\section{Correlation between X-ray intensity and interferometer phase shift measurements}

An Oxford Instruments $50 \mathrm{kV}$ tube (XTF 5011) with a Mo target and a maximum anode current of $1 \mathrm{~mA}$ was mounted with its exit window $7.0 \pm 0.5 \mathrm{~mm}$ above the CZT crystal. A $0.6 \mathrm{~cm}^{3}$ ionization chamber (N.E. Technology 2571A) was used to roughly estimate the air kerma at the sample position due to the X-ray flux to enable future rough comparisons in terms of X-ray intensity. The measurement neglects the difference in secondary emissions, air ionization, scattering and absorptions due the surroundings of the ionization chamber during measurement not being the same as the CZT mounting. As expected, the measured kerma at $50 \mathrm{kVp}$ increases linearly from background level at zero anode current to $0.05 \pm 0.01 \mathrm{~Gy} / \mathrm{s}$ at $1 \mathrm{~mA}$.

Fig. 5 shows the average phase shift measured when switching the applied bias voltage across the CZT crystal from $0 \mathrm{~V}$ to the value indicated as well as a linear fit through the data. The phase shift clearly increases with increasing bias voltage relative to the zero value. Using the laser wavelength $\lambda=1550 \mathrm{~nm}$ and $n_{0}=2.72$ (Ref. 19) in equation (2) results in a reduced value of $r_{41}=$ 
$5.6 \pm 0.5 \times 10^{-12} \mathrm{~m} / \mathrm{V}$. This reduction could originate in the wavelength dependence of the electro optic coefficient, however it may also be caused by the fact that the probing IR laser itself causes space charge to build up in the device and the presented interferometer system will only present an average effect over the sample cross section probed (folded by the beam intensity profile incident on the photodiode). Subsequently, the systematic deviation from predicted linear increase of average phase shifts with applied bias voltage could also be caused by a phase shift distribution that includes shifts in both directions. This is qualitatively in agreement with the Pockel's imaging data which indicates field enhancement near the cathode and lower fields near the anode. The charge trap and hence defect distribution in the material will affect the extent of the aforementioned space charge build-up and this is therefore directly related the sample quality. However the data shown in Figure 5 agrees with a $V^{2}$ dependence, which has also been reported by Shwartz et al (Ref. 8) for refractive index (i.e. phase shift) changes in Vanadium doped CZT.

Similarly Fig. 5 shows a linear increase of phase shift with increasing X-ray tube current (at 500 $\mathrm{V}$ applied bias at the CZT crystal) for tube current values above $0.3 \mathrm{~mA}$, at a rate of $1.58 \pm 002$ rad per mA. The phase shifts extracted at lower tube currents, i.e. low dose rates, exhibit an unexpected behavior that needs further investigation and seems to lead to an apparent offset in the linear section of the observed phase shift vs tube current relationship. A possible qualitative explanation for this behavior may be that at low dose rates, the laser beam is covering sample areas where the electric field increases (near the cathode) and decreases (near the anode) under irradiation. The overall effect is that the laser light undergoes phase shifts in opposite directions in the different areas and the overall shift observed appears reduced compared to the expected 
linear increase. At higher irradiation levels one contribution may dominate and the system approaches linear behavior.

\section{Conclusion}

The data acquired with the collimated IR laser suggests that the spatial space charge distribution profile produced by illumination induced free charge carriers does not exclusively depend on the location of the free charge carrier generation. The results demonstrate that the direct measurement of the phase shift of polarized light transmitted through CZT under X-ray irradiation can be used - following careful calibration and improved vibrational stability - to deduce deposited X-ray energy per time in the detector volume.

Acknowledgments

S. Parsons acknowledges financial support through STFC grant nr. ST/K005669/1. 
Figure 1: Figure 1: Schematic of the Pockels imaging setup used to acquire images under IR laser illumination. The CZT sample is mounted on a height adjustable stage. The image in the inset on the right shows the intensity image acquired of the sample between crossed polarised with Cathode at the top at $500 \mathrm{~V}$ applied bias comparing laser on and off images.

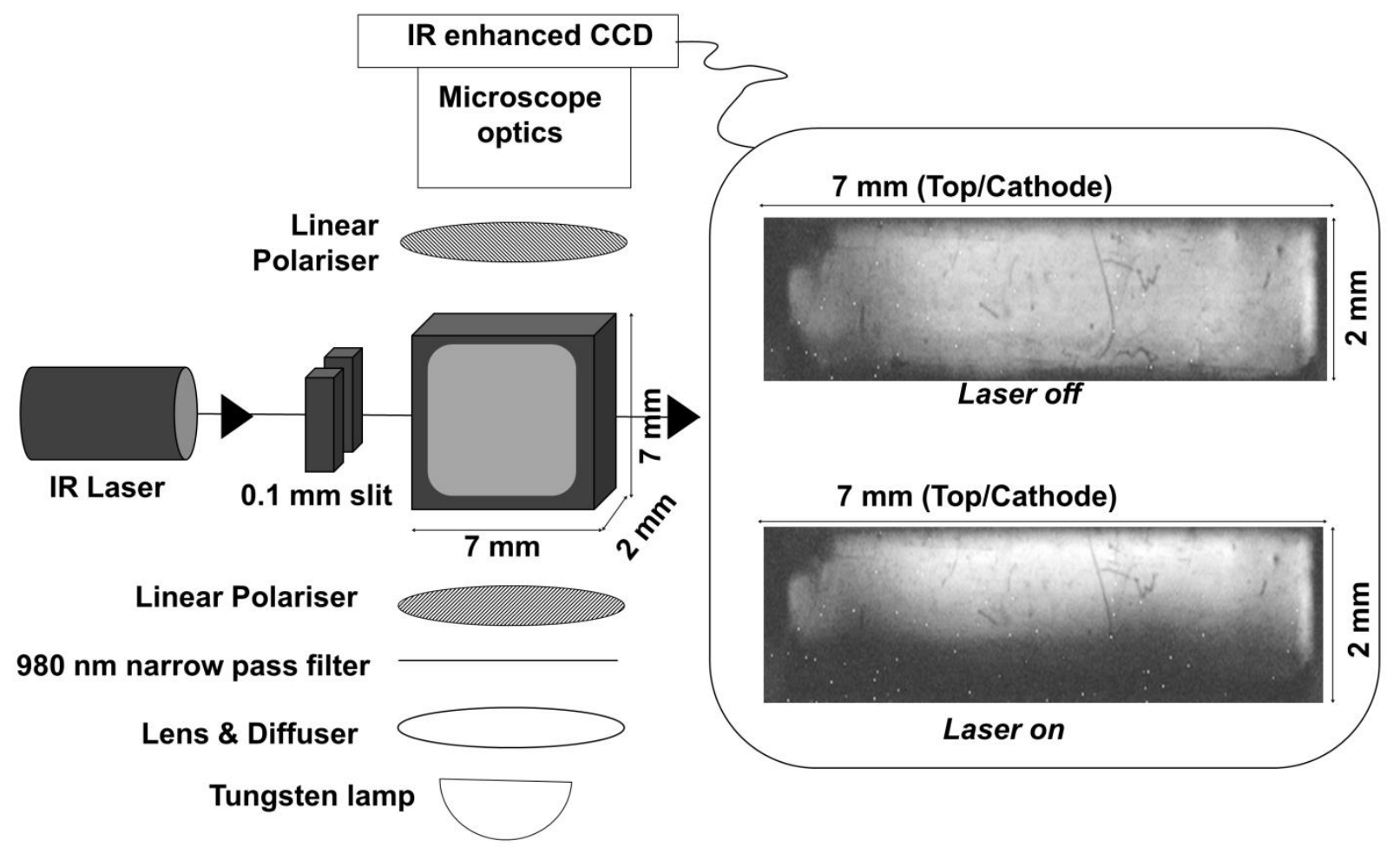


Figure 2: Pockels image intensity between the two electrodes (at position $0 \mathrm{~mm}$ and $2 \mathrm{~mm}$ ). a) at $+500 \mathrm{~V}$, i.e. cathode at $0 \mathrm{~mm}$ (grey) and $-500 \mathrm{~V}$, i.e. Cathode at $2 \mathrm{~mm}$ (black), without IR laser illumination (solid line) and under full IR laser illumination (dashed line).b) at $+500 \mathrm{~V}$, i.e. cathode at $2 \mathrm{~mm}$ position as a function of incident height in $0.2 \mathrm{~mm}$ steps over $6 \mathrm{~mm}$ range.

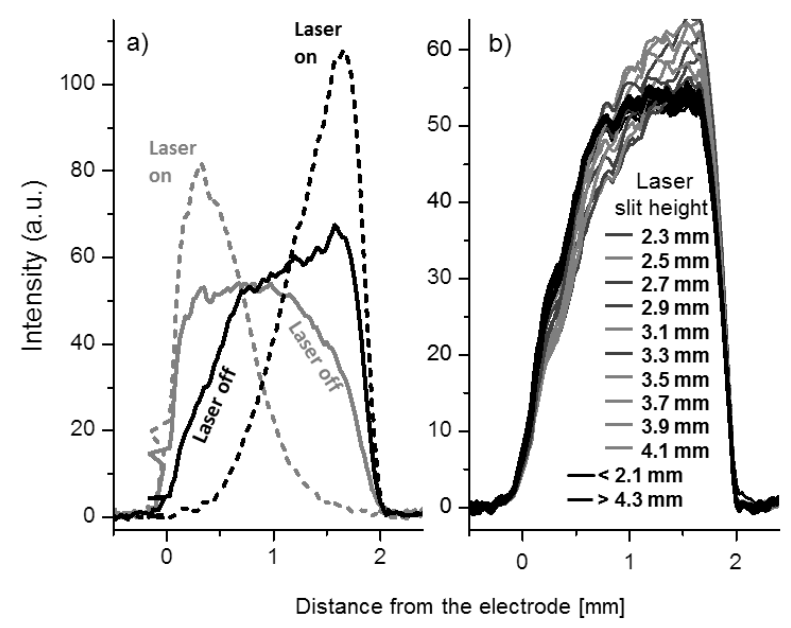

Figure 3: Schematic diagram of the Interferometer set-up

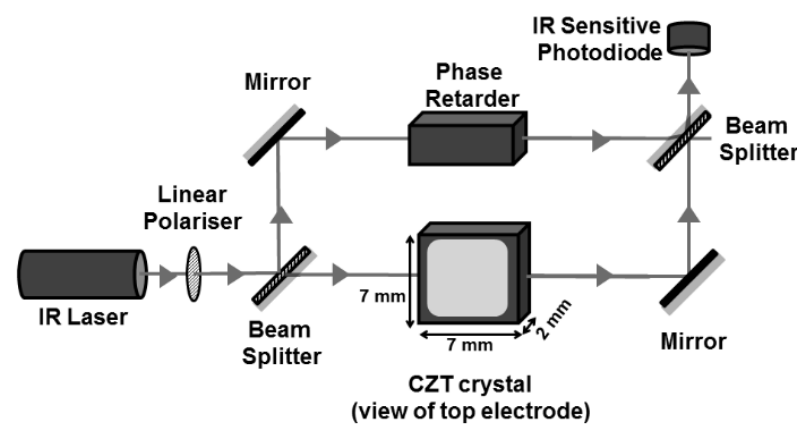


Figure 4: Schematic diagram of a typical oscilloscope trace analysed.

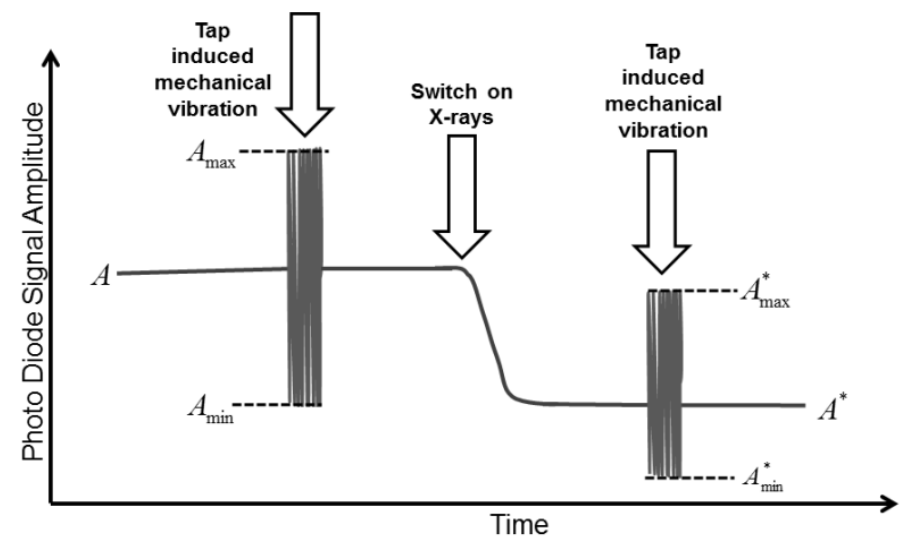

Figure 5: Observed phase shift (averaged over 10 readings per point shown) when changing the applied bias from $0 \mathrm{~V}$ to the value indicated on the bottom axis (open circles) without X-ray irradiation and as a function of changing X-ray Anode current from $0 \mathrm{~mA}$ to the value indicated at the top axis, at a fixed applied bias Voltage of $500 \mathrm{~V}$ (solid squares).

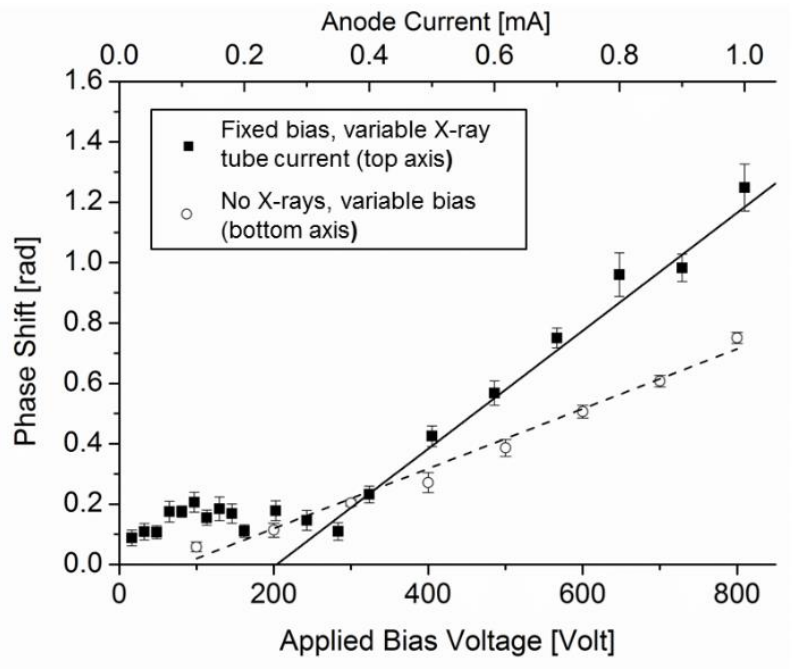

${ }^{1}$ T. E. Schlesinger, J. E. Toney, H. Yoon, E. Y. Lee, B.A. Brunett, L. Franks, and R. B. James, Materials Science and Engineering 32, 103 - 189 (2001).

${ }^{2}$ J. Grindlay, J. Hong, B. Allen, S. Barthelmy, and R. Baker, Nuclear Instruments and Methods in Physics Research A 652, 671-673 (2011). 
${ }^{3}$ C. Oldorf, Journal of Physics: Conference Series 375, 042022 (2012).

${ }^{4}$ Y. A. Boucher, F. Zhang, W. R. Kaye, and Z. He, IEEE Transactions on Nuclear Science 60(2), 1086-1093 (2013).

${ }^{5}$ T. Kurosawa, H. Iwase, and N. Saito, Journal of Nuclear Science and Technology 51(5), 730734 (2014).

${ }^{6}$ G. Yang. A. E. Bolotnikov, G. S. Camarda, Y. Cui, A. Hossain, H.W. Yao, and R. B. James, Journal of Electronic Materials 38 (8), 1563 - 1567 (2009).

${ }^{7}$ A. Yariv, Optical Electronics in Modern Communications, 5th Edition, Oxford University Press (1997).

${ }^{8}$ S. Shwartz, M. Segev, S. Berger, E. Zolotoyabko, and E. El-Hanany, Physical Review B 79, $193202(2009)$.

${ }^{9}$ G. Prekas, P. J. Sellin, P. Veeramani, A. W. Davies, A. Lohstroh, M. E. Özsan, and M. C. Veale, J. Phys. D: Appl. Phys. 43, 085102 (2010).

${ }^{10}$ A. L. Washington II, L. C. Teague, M. C. Duff, A. Burger, M. Groza, and V. Buliga, J. Appl. Phys. 111, 113715 (2012)

${ }^{11}$ A. L. Washington II, L. C. Teague, M. C. Duff, A. Burger, M. Groza, and V. Buliga, Journal of Electronic Materials 111, 2875-2879 (2012)

${ }^{12}$ A. Langley, MPhys Disseratation, University of Surrey, UK (2013)

${ }^{13}$ K. A. Nelson, N. Edwards, M. J. Harrison, A. Kargar, W. J McNeil, and D. S. McGregor, Nuclear Instruments and Methods in Physics Research A 620, 363-1367 (2010).

${ }^{14}$ K. A. Nelson, J. A. Geuther, J. Neihart, T. A. Riedel, R. A. Rojeski, P. B. Ugorowski, and D. S. McGregor, Nuclear Instruments and Methods in Physics Research A 680, 97-102 (2012). 
${ }^{15}$ K. A. Nelson, J. A. Geuther, J. Neihart, T. A. Riedel, R. A. Rojeski, J. L. Saddler, A. R.

Schmidt, and D. S. McGregor, Applied Radiation and Isotopes 70, 1118-1120 (2012).

${ }^{16}$ K. A. Nelson, K. R. Arpin, J. A. Geuther, J. L. Neilhart, T. A. Riedel, R. A. Rojeski, P. B.

Ugorowski, and D. S. McGregor, IEEE Nuclear Science Symposium Conference Record, R20-1 (2011).

${ }^{17}$ Y. Xu, W. Jie, P. J. Sellin, W. Liu, G. Zha, P. Veeramani, and C. Mills, J. Phys. D: Appl. Phys. 42, 035105 (2009).

${ }^{18}$ G. Yang, A. E. Bolotnikov, G. S. Camarada, Y. Cui, A. Hossain, K. H. Kim, and R. B. James, "Electric field distributions of Cadmium Zinc Telluride (CZT) detectors, presented at the 2009 SPIE Optics and Photonics conference.

${ }^{19}$ M. A. Quijida, and R. Henry, Proc. SPIE 6692. Cryogenic Optical Systems and Instruments XII, 669206 (2007).

${ }^{20}$ A. Cola, I Farella, N. Auricchio, and E. Caroli, Journal of Optics A: Pure and Applied Optics 8, S467-S472 (2006). 